\title{
Visually presented verbal stimuli by assembly language on the Apple II computer
}

\author{
JOHN K. ADAMS \\ New York University, New York, New York
}

\begin{abstract}
A method for programming real-time experiments employing visually presented verbal stimuli is described for the Apple II computer. Applesoft BASIC is used to load the stimuli and write the data file, but assembly language controls all aspects of the experimental trials. The method is illustrated for a lexical decision experiment; however, it readily extends to semantic priming, recognition, sentence verification, and single- or multiple-line reading experiments. The method maximizes both precision and stimulus capacity.
\end{abstract}

Many experiments employing visually presented verbal stimuli require precise control of a succession of events and accurate measurement of reaction times. Continuous lexical decision (Rubenstein, Garfield, \& Millikan, 1970) and semantic priming (e.g., Neely, 1977) are two salient examples. In continuous lexical decision, the onset of the target stimulus marks the beginning of a latencymeasurement period that is terminated by the subject's response. The response marks the beginning of an interstimulus interval (ISI) after which the next stimulus is presented. In semantic priming, the prime stimulus is presented first. After its offset, a blank-screen interval occurs. Following this, the target stimulus is presented. The interval between prime onset and target onset, the stimulus onset asynchrony (SOA), must be precisely controlled.

The need for precision, however, is not limited to these paradigms. Experiments in sentence verification (Collins \& Quillian, 1969) and reading (e.g., Glanzer, Dorfman, $\&$ Kaplan, 1981) require accurate measurement of reaction times and strict control of experimental events. Finally, recognition memory experiments (e.g., Hockley, 1982) often require exact pacing.

In recent years, many of these experiments have been run on microcomputers. The objective has been to obtain millisecond accuracy both in latency measurement and in the control of experimental events. Two programming methods have been used. With the first method, all aspects of the experimental trial are conducted in a high-level language such as BASIC. The high-level program is run under interpretation or compiled into machine code which is executed directly. Under interpretation, the execution time for a given program line can be quite long and variable (Dlhopolsky, 1983). When a program is compiled,

This work was supported in part by BRSG Grant RR-07062 awarded by the Biomedical Research Support Grant Program, Division of Research Resources, National Institute of Health. Special thanks are due to Doris Aaronson, Murray Glanzer, Brian Watts, and two anonymous reviewers for their valuable comments on an earlier draft of this paper. The author's mailing address is: Department of Psychology, New York University, 6 Washington Place, 8th Floor, New York, NY 10003. execution speed is improved. However, compiled programs are not optimally efficient. Execution speed still might not be great enough to provide sufficient precision in time-critical operations such as the monitoring of response ports.

The desire for accuracy in these operations has led some experimenters (e.g., Rayfield \& Carney, 1981) to use a second programming method with which assembly language subroutines are called from a high-level program for one or more operations deemed time-critical. Assuming that the subroutines are well-written, this method results in millisecond precision for these operations. However, this method may not provide millisecond precision for the remaining operations because they still are handled by the less efficient high-level program.

\section{APPLESOFT BASIC COMMANDS AND REAL-TIME PRECISION}

Tests were carried out to determine the effect on realtime precision of using high-level commands during the experimental trial. Interest was focused on the execution speed of Applesoft BASIC commands in the Apple II+ computer. The commands chosen for testing were those that would still have to be executed, in the previouslymentioned applications, if the second programming method were used. Specifically, it was assumed that assembly language subroutines would be called for response monitoring (coupled with latency measurement) and for interval timing (such as for the ISI).

The method of testing was as follows. Each Applesoft BASIC command was placed alone in a loop that executed 1,000 times. The test program read a firmware clock (accurate to $1 \mathrm{msec}$ ) immediately before and immediately after the loop iterations. Each program was run several times. An empty-loop version was also run. Parallel tests were conducted using interpreted and Microsoft-TASC compiled code. The commands of interest were written to be maximally efficient (e.g., in compiled versions, integer operations were specified wherever possible).

For each command, the total time for 1,000 executions was obtained by subtracting the total time for 1,000 empty- 
loop iterations. The time to perform each BASIC operation once was obtained by dividing the adjusted total time by 1,000 . (Since 1,000 iterations occurred between readings of a clock that is accurate to $1 \mathrm{msec}$, the error of measurement for a given execution time is plus or minus $.001 \mathrm{msec}$.) The execution times for the commands are listed in Table 1. Compilation increases execution speed, but certain commands require more than $1 \mathrm{msec}$ to execute even if compiled. Printing a string of characters takes considerable time, and the time increases very quickly as the length of the string increases.

The compiled-code measurements provide a basis for evaluating the maximum real-time accuracy of the second programming method as it is applied to the experiments outlined earlier. (The performance of interpreted code is not considered further.) In any application using the second method, one or more blocks of BASIC operations must be executed during a trial. A block is defined as a sequence of BASIC commands that is executed between two consecutive periods of assembly language control. In order to maintain real-time accuracy at the millisecond level, a given block must execute in less than $1 \mathrm{msec}$. If it does not, the real-time flow of experimental events is disrupted and accuracy is compromised. If execution time in excess of $1 \mathrm{msec}$ represents the real-time error for a given block, then the sum of these excess times across all blocks of the trial is the total real-time error attributable to the high-level program for that trial.

For continuous lexical decision (and its programming equivalent, recognition), it is assumed that the stimulus is 10 characters long (including leading blanks for centering), and that a one-line text window is used. There are two blocks: stimulus-on and post-response. The former requires $6.93 \mathrm{msec}$ : loop iteration + print 10 characters + call a latency routine. The latter requires 6.22 msec: clear one line + store response + calculate and store latency + call ISI routine. The total real-time error per trial is $(6.93-1.00)+(6.22-1.00) \mathrm{msec}$ or $11.15 \mathrm{msec}$. For semantic priming, the stimulus and text-window assumptions are the same, but there are four blocks: prime-on, prime-off, target-on, and post-response. These blocks require $6.93,2.56,6.73$, and $6.22 \mathrm{msec}$, respectively, giving a total real-time error per trial of $18.44 \mathrm{msec}$. For sentence verification, it is assumed that a 30-character stimulus is presented and that a one-line text window is used. There are two blocks: stimulus-on and post-response. The blocks require $\mathbf{1 5 . 7 2}$ and 6.22 msec, respectively, giving a total real-time error per trial of $19.94 \mathrm{msec}$. For a multiple-line reading experiment, it ; is assumed that the stimulus is 60 characters $\left(1 \frac{1}{2}\right.$ lines) long and that a two-line text window is used. There are two blocks: stimulus-on and post-response. They require 29.72 and $6.87 \mathrm{msec}$, respectively, giving a total realtime error per trial of $34.59 \mathrm{msec}$.

It is clear that performing operations in BASIC, as required by either programming method, disrupts the experimental sequence even if the program is compiled. The major source of disruption involves presentation of the stimulus.

\section{AN ASSEMBLY-LANGUAGE METHOD}

Program accuracy would be maximized if the entire experimental trial were carried out by a well-written assembly language program. For this method to work, however, two different programs must be able to interact: A highlevel program must store stimulus characters in an array that can be used by an assembly language program, and an assembly language program must create a data array that can be used by the high-level program. Fortunately, this can be done quite easily. A BASIC program can store stimulus characters sequentially in free RAM. An assembly language program can use this array as a source of stimulus characters and can create a data array of the same type for use by the BASIC program.

In this article, I describe a method for conducting the trials of experiments employing visually-presented verbal stimuli completely within assembly language. In most applications, the method provides millisecond precision for program operations. Also, memory use is very efficient. This efficiency is attained in three ways. First, a chaining technique is used (in which one BASIC program runs another) to minimize the amount of BASIC code in memory at any one time. Second, the program that controls the trials is placed in bank-switched firmware RAM so that it can be as elaborate as necessary without reducing stimulus or data storage capacity. Third, the stimulus and data arrays are completely independent. Therefore,

Table 1

Execution Time for Selected Appleson BASIC Commands

\begin{tabular}{llrr}
\hline \multicolumn{1}{c}{ Operation } & \multicolumn{1}{c}{ Command } & \multicolumn{2}{c}{ Time (msec) } \\
\cline { 3 - 4 } Loop iteration & FOR I=1 TO N . . NEXT & 1.27 & Compiled \\
Print 7 characters & PRINT S\$(I) & 7.24 & .20 \\
Print 10 characters & $"$ & 8.54 & 4.62 \\
Print 30 characters & $"$ & 17.20 & 5.93 \\
Print 60 characters & CALL 768 & 32.38 & 14.72 \\
Call (with Return) & CALL -936 & 3.92 & 28.72 \\
Clear 1-line window & " & 5.19 & .80 \\
Clear 2-line window & R(I)=PEEK(6) & 5.84 & 1.76 \\
Store response & RT(I)=(PEEK(7)*K)+PEEK(8) & 4.93 & 2.41 \\
Calculate latency & & 8.57 & 1.12 \\
\end{tabular}


the stimulus array can initially occupy virtually all of free RAM. As data are collected, they are saved in the previously used portion of the stimulus array.

\section{Applicability}

The method is applicable to a wide variety of experiments employing visually-presented verbal stimuli. However, the stimuli must consist entirely of ASCII characters, and the primary text pages of screen refresh memory must be used. The method does not extend to experiments using graphics or color.

The method results in precisely timed program operations; however, real-time precision depends on more than this. The video screen is an important source of error. One problem is that screen phosphors take time to decay after the stimulus is removed by the program. This effect can be minimized by conducting the experiment in a bright room and using minimum screen brightness. A more serious problem is that the $60-\mathrm{Hz}$ screen refresh cycle operates independently of the program, resulting in a random delay (up to $16 \mathrm{msec}$ ) of stimulus onset. (Reed, 1979, discusses the problem and its solutions.) If a conventional screen is used, the error component cannot be removed from the trial. However, the random component can be relegated to the period immediately prior to stimulus onset by synchronizing the programmed stimulus onset with the screen cycle. Synchronization is easily accomplished in assembly language. The appropriate code is given later.

\section{Equipment}

The method was developed for an Apple II + or IIe (64K) computer with Applesoft BASIC in ROM and DOS 3.3 disc operating system. However, the method should prove adaptable to other (64K) 6502 machines. For the illustrative programs, I assume that the computer is an Apple II + (with Apple Language Card or equivalent to extend capacity to $64 \mathrm{~K}$ ), and that a Mountain hardware clock is resident in Slot 4. ${ }^{1}$ A Microsoft-TASC compiler is used to compile the BASIC code. ${ }^{2}$ (A compiler is not required. A procedure for using the method under interpretation is outlined later.) The Apple 6502 Assembler/Editor is used to create the assembly code.

The three single-bit inputs of the Apple II + game I/O connector (locations \$C061-\$C063 hexadecimal) are used as response ports. A response board is used that consists of three microswitches (corresponding to left, right, and start keys). Each switch is wired with a pull-up resistor $(10-100 \mathrm{k} \Omega)$ that keeps the value in the corresponding port high as long as the switch is open. When the subject makes a response and closes the switch, the corresponding port is pulled low. (This configuration is somewhat arbitrary and is based on the resting value of the inputs in the Apple II + . Minor program changes will be required to accommodate other configurations.) The Apple keyboard also can be used to collect responses. A method for doing this is outlined later.

\section{Latency and Interval Timing}

Response latencies are obtained in the following way. During the response monitoring process, changes (or "ticks") in the 1-msec output buffer of the firmware clock are counted. Zero-page locations are incremented as ticks occur. After a response is detected, the values in the zeropage locations are stored in the data array. Because only one clock location is examined, the code required is minimal (and consequently very fast). Also, the procedure is independent of the coding format used in the clock's 1-msec output buffer. The program determines only whether or not the value in the buffer has changed; it does not interpret the value. Therefore, it does not matter whether the coding format of the particular buffer is binary or binary-coded decimal. The timing of programmed intervals such as the ISI is also accomplished by counting ticks. Software clock routines can be substituted for the firmware ones. Interrupts are not used.

\section{Overview of the Method}

Four illustrative programs are written for a continuous lexical decision experiment. Two are written in BASIC; they operate in a chain and are compiled separately using default parameters. Two are written in assembly language: One resides on Page $3(\$ 300-\$ 350)$ and consists entirely of subroutines that facilitate the use of firmware RAM; the other resides in the firmware RAM (\$E000-\$E144). It runs all trials of the experiment and is referred to as the trials program. ${ }^{3}$

An experiment begins by running the first BASIC program (see Appendix A). The program performs in order the following operations: It loads the Page-3 program, calls one of the Page- 3 subroutines to copy the Monitor ROM into the firmware RAM, loads the trials program into the firmware RAM, obtains subject parameters, establishes zero-page values for the trials program, creates the stimulus array (working from a lower RAM address to a higher RAM address), and calls the trials program. When control is returned to the first BASIC program, it writes a temporary data file that is essentially an image of the data array created by the trials program. The second BASIC program (see Appendix B) is then run. It uses conventional arrays and creates the final data file from the temporary file.

\section{INPUT AND OUTPUT}

The stimulus file is a text file. For lexical decision, the stimulus file consists of a series of ID number-stimulus sets with the ID number (I) on one line and the stimulus string (S) on the following line. The first line of the file indicates the total number (N) of ID-stimulus sets in the file. Thus the stimulus file has the general configuration:

$$
\mathrm{N} / \mathrm{I} 1 / \mathrm{S} 1 / \mathrm{I} 2 / \mathrm{S} 2 / \ldots / \mathrm{IN} / \mathrm{SN} /
$$

where a slash indicates a carriage return. The order of the sets is determined prior to the experiment. 
The permanent data file consists of sets in which a given ID number is followed by response code $(R)$ which in turn is followed by the reaction time (T) in msec. Thus the data file has the following general configuration:

$$
\text { N/I1/R1/T1/I2/R2/T2/ . . /IN/RN/TN/ }
$$

\section{PROGRAM OPERATIONS}

The Page-3 program. The Page-3 program (see Appendix C) consists of three subroutines; the first two are called by the first BASIC program. The first subroutine (CPMON) copies the Monitor ROM into the firmware RAM. This allows Monitor subroutines to be called, if necessary, by the trials program. The Monitor ROM is not normally available when the firmware RAM is enabled. The second subroutine (SWITCH) enables the firmware RAM, establishes a reset vector so that the trials program can be aborted, if necessary, without turning off the machine, and jumps to the trials program. When the trials are completed, the subroutine disables the firmware RAM and returns control to the first BASIC program. The third subroutine (RESET) receives control upon reset, by means of the vector established by SWITCH; it simply disables the firmware RAM and jumps to the Monitor reset routine.

Initial operations. The first BASIC program is listed as Appendix A. Lines 90-120 obtain subject parameters. Since the illustrated task is lexical decision, handedness is important. The dominant hand is used to press a YES button indicating the item is a word; the nondominant hand is used to press a NO button indicating the item is not a word.

It is assumed that an independent variable in the experiment is the length of the ISI. At Line 150, the number of 50-msec blocks of ISI is POKEd to the zero page for use by the trials program. The value illustrated results in a 500 -msec ISI.

One of the features of the Microsoft-TASC compiler is that, when the compiled program is run, the address of the highest RAM byte used is placed at $\$ 32-\$ 33$. Line 160 obtains this address and calculates the lowest free RAM byte (FR). This value of FR represents the address of the first byte of the data array. At Line 170 , pointers for this address are calculated. Then FR is increased by 64 bytes. At Lines 190-200, pointers for the start of the stimulus array are calculated and POKEd to the zero page. Starting the stimulus array slightly higher in memory than the data array insures that stimuli which have not yet been presented will not be overwritten with data.

Next, the stimulus file is opened. The number of ID-stimulus sets (here termed L) is input. For each set, the ID number and stimulus are input. Any practice items appear first in the file and are identified as such by a zero ID value. A count of practice items is made as the file is read. After a stimulus is read, its length is found. Then, based on the length, the number of leading blanks (B) required to center the stimulus is determined.

At Line 310, the length, including leading blanks, of a given stimulus is POKEd into the array first. Any lead- ing blanks are POKEd using the ASCII value for a blank with the high bit set (i.e., the ASCII value plus 128). This is required for all characters presented on the Apple's screen. Note that the centering can be performed by the trials program. This increases stimulus capacity because blanks are not placed in the array. Because the arrays are of high capacity, however, the simpler procedure is adequate for most applications. At Line 330, the ASCII value for each stimulus character is obtained and POKEd into the array with the high bit set.

The top 400 bytes of free RAM (decimal 38001-38400) are reserved as a string-operations buffer. If the stimulus file is so large that the array extends into the buffer, the program will abort (Line 260). Garbage collection is forced after each array element has been stored (Line 340) to confine string operations to the buffer.

Assuming no practice items, the number of experimental (nonpractice) stimuli is $N=\mathrm{L}$. In Lines $430-440$, the trials count and pointers for the data array are POKEd to the zero page. Finally, control is transferred to the trials program in firmware RAM by a CALL to the Page-3 subroutine SWITCH.

Experimental trials. An assembler listing of the trials program is given in Appendix C. The program uses the primary text pages of screen refresh memory $(\$ 400-\$ 7 F F)$ to present the stimuli. Since the Apple's screen is memory mapped, storing the ASCII value of a character (plus 128) at a specific location within this range will cause the character to appear at a specific place on the screen. Subsequently storing a blank at the same location will cause the character to be cleared from the screen.

In the illustrated application, the memory area corresponding to Line 10 of the screen is used. Column 12 of this line (location $\$ 534$, labeled SCR) is used as the starting column for each stimulus in order to minimize the number of leading blanks required for centering. (For individual applications, see The Apple II Reference Manual, 1981, pp. 14-16.)

The program first prints a logo on the screen. This is a series of asterisks that remains on the screen until the trials begin. (The logo is useful in adjusting the subject's monitor and in referring to the future location of the stimuli during instructions.) Next, a START button subroutine executes until the start port is pulled low by a key press. When this happens, the screen is cleared by subroutine CLR. (This subroutine stores 15 blanks on Line 10 of the screen, starting at Column 12. Because a minimum number of blanks is stored, it is much faster than the Monitor's HOME routine.) After returning from START, a 5-sec pause ensues with a JSR to subroutine INT. (The Y-register contains the number of 50-msec blocks desired.) The pause allows the subject time to reach the RIGHT and LEFT response keys before the first stimulus is presented.

The trials loop (Lines 69-147) is then entered. The program checks to see that the subject is not pressing a response key by a JSR to the NOPRess subroutine which executes until both response ports are high. Then the number of trials remaining is checked and decremented. At 
label STIMON each leading blank and stimulus character is placed on the screen. Then a JSR is executed to a subroutine that UPDATEs the stimulus pointers.

Next, the program reads the clock's 1-msec output buffer and stores the value. The low and high bytes of the number of ticks are zeroed. The response ports are then checked (label PCHECK). The Y-register contains 1 when the RIGHT port is checked and contains 0 when the LEFT port is checked. If no response is detected, the clock is read again, and the new value is compared to the stored value. If the values are the same, then no tick has occurred and the response ports are rechecked. If they are different, then the new clock value is stored and the number of ticks is incremented. If the number of ticks has reached an arbitrary maximum value, a nonresponse is recorded at NORESP.

When a response is detected, a branch to label RTCHK occurs. Any keybounce, or anticipatory responding on the part of the subject, is isolated. The latency is checked to see that it exceeds an arbitrary minimum value. If it does, the response is accepted. If it does not, a branch to a "timed nopress'" (TNOPR) occurs. The counting of ticks continues until the high bit is set in both response ports, indicating either that the switch has bounced open or that the subject has stopped pressing the key. When both ports are high, another response is sought. If keybounce produced the original response, and the key is still bouncing, then another response will soon be detected and another branch to TNOPR will occur. The process will continue until the bouncing stops. (The TNOPR routine insures that keybounce will not affect the accuracy of latency measurement.)

After an acceptable response, the screen is cleared and the data are stored in the data array by subroutine SAVDAT. First, the response is stored, followed by the two latency bytes. The data pointers are then updated. Finally, a timed ISI ensues. (Subroutine INT times the interval. If a 50-msec block size is used, as in this application, the maximum timed interval possible is $12.75 \mathrm{sec}$. Larger and smaller block sizes result in larger and smaller maximum intervals, respectively.) When all trials are completed, an RTS returns control to the first BASIC program (via SWITCH on Page 3).

Synchronization. On the Apple IIe, synchronization of the experimental program with the screen refresh cycle is easily accomplished since the vertical blanking signal (VBL) is already available at location \$C019. On the Apple II + , the signal must be made available with a minor hardware modification (see Reed, 1979, for details). The modification requires that one of the three single-bit inputs be used for the VBL, leaving only two inputs for keypresses. To preserve the three-key arrangement, the start key can be wired to either the left or right response input. The result is that one input handles both a response and the start keypress. This arrangement should not prove troublesome in most applications. Assuming that the VBL is available at location LOC, and that LOC is pulled low by the VBL as on the Apple IIe, the following code is executed with a JSR, just before stimulus presentation, to achieve synchronization.

$\begin{array}{llll}\text { SYNC } & \text { BIT } & \text { LOC } & \\ & \text { BMI } & \text { SYNC } & \text {;AWAIT VBL ONSET } \\ \text { SYNC1 } & \text { BIT } & \text { LOC } & \\ & \text { BPL } & \text { SYNC1 } & \text {;AWAIT VBL OFFSET } \\ & \text { RTS } & & \end{array}$

Keyboard Responses. The Apple keyboard can also be used to collect responses in place of the game I/O connector. The following code is used.

$\begin{array}{lccl}\text { KBCHECK } & \text { BIT } & \$ C 000 & \text {;CHECK KEYBOA } \\ & \text { BMI } & \text { RSAVE } & \text {;BRANCH IF RESPO } \\ & \text { JMP } & \text { KBCHECK } & \\ & \cdot & \cdot & \\ \text { RSAVE } & \text { BIT } & \$ C 010 & \text {;CLEAR STROBE } \\ & \text { LDA } & \text { \$CO00 } & \text {;GET RESPONSE } \\ & \text { STA } & \text { TEMP } & \text {;SAVE }\end{array}$

A NOPRess subroutine is not possible when the keyboard is used. Therefore, it is recommended that the instruction

\section{LOOP $\quad$ BIT $\$ C 010 \quad$;CLEAR STROBE}

be used to cancel any spurious keypress made by the subject during the ISI. Like the game-port routine, the keyboard routine executes very quickly. However, obtaining responses from the keyboard will probably compromise the accuracy of obtained latencies because the keys do not operate as efficiently as microswitches.

Practice Trials. If there are practice trials, lines 380410 of the first BASIC program (Appendix A) apply. The number of practice items is POKEd at Line 380. (This is analogous to Line 430 for the experimental trials.) At Line 390, the starting address of the data array is POKEd. The trials program is then called to run the practice trials. (Since the original data pointers are POKEd again just prior to the experimental trials, the practice data are overwritten.) The final data count (N) is adjusted to exclude the practice items.

Output of Raw Data. After the experimental trials are completed, the first BASIC program continues at Line 460 (Appendix A). A temporary file is opened on the floppy disc to receive the raw data. (This file is overwritten each time a subject is run.) Parameters are saved first, then the raw data are output to the temporary file in the order that they were entered into the array by the trials program.

Making the Permanent Data File. After the raw data are output, the second BASIC program is run. This program is listed in Appendix B. First, conventional arrays are established for stimulus ID number, response (R), and reaction time (RT). Then the raw data are input. A single latency value is calculated from the two latency bytes of a given trial. Lines 140-150 illustrate a response conversion for left-handed subjects. 
After the raw data are input, the program obtains ID numbers. Items for which data are not to be retained (e.g., header items) are identified by ID number, and the final data count (N) is reduced as they are encountered.

After the ID numbers are input, the permanent data file is created. The final data count is entered as the first line of the file. The data are then output from the arrays, skipping any that are not to be saved. When the file is completed, a summary program is run.

Running Under Interpretation. Even though input and output are slowed considerably, it is possible to run the BASIC programs under interpretation. The only difficulty lies in determining the first free RAM byte (i.e., the initial value of FR). The following procedure is recommended. First, a stimulus file is prepared which contains one practice item and one experimental item. Next, the first BASIC program is loaded and the following temporary lines are entered.

$$
\begin{array}{ll}
160 & \text { FR }=36000 \\
565 & \text { END }
\end{array}
$$

The program is saved, memory is cleared, and the following line is typed in and run.

\section{FOR I $=2100$ TO 38000: POKE I,0: NEXT}

The line will place zero's in virtually all of free RAM. Next, the modified version of the first BASIC program is run using the short stimulus file. After the responses are made and the run is completed, the Monitor is entered and, using the " $L$ " command, memory is examined from location $\$ 800$ upward. Listing is continued until an uninterrupted sequence of zero's (BRK opcodes) is encountered. The lowest address of this sequence is the lowest free byte of RAM. After adding 100 or so to the decimal equivalent of this value, the initial value of FR is permanently set in the first BASIC program (Line 160) and the other temporary line is deleted. Any BRUN commands are replaced with RUN commands in both BASIC programs.

\section{OTHER PARADIGMS}

Single-Word Recognition and Semantic Priming. The illustrated programs can be adapted to single-word recognition experiments without modification. Semantic priming experiments can be accommodated quite easily. The stimulus file should be configured as

$$
\text { N/I1/P1/T1/I2/P2/T2/ . . /IN/PN/TN/ }
$$

where $I, P$, and $T$ are the ID number, prime stimulus, and target stimulus, respectively.

The first BASIC program would POKE the length and characters of the prime and target for a given trial consecutively in the stimulus array. On a given trial, the trials program would present the prime, update the stimulus pointers, present the target, and update the stimulus pointers again. All timed intervals would be controlled by subroutine INT. Finally, in obtaining ID numbers for the data file ${ }_{3}$ the second BASIC program would skip over stimulus entries in multiples of two.

Multiple-Word Single-Line Paradigms. If the longest stimulus used in a sentence recognition, sentence verification, or reading experiment is 40 characters or less, including spaces and punctuation, the programs can be adapted easily. Since these experiments usually do not require that the stimuli be centered, the calculating and POKEing of leading blanks can be eliminated. However, if indentation or punctuation is used, characters should be obtained from the stimulus file using the GET command, rather than the INPUT command, to prevent character omissions.

In reading experiments, the START button would also serve as an ADVANCE button. The program would collect a dummy response from the MID port (e.g., '"2') on each reading trial. If comprehension questions are presented during the experiment, they can be signaled (to both the subject and the trials program) by the initial stimulus character (e.g., an asterisk). The trials program would check the initial character of each stimulus to see if it is an asterisk and, if it is, branch to a subroutine to examine the LEFT and RIGHT ports, rather than the MID port.

Multiple-line Experiments. If the stimulus can be longer than one screen line, the changes to the programs are more extensive. The stimulus file will also have a different configuration. A given stimulus will consist of an ID number (I) followed by $J$ stimulus lines $(\mathrm{L} 1, \mathrm{~L} 2, \ldots, \mathrm{LJ})$. The general configuration of the file is

$$
\mathrm{N} / \mathrm{I} 1 / \mathrm{L} 1 / \mathrm{L} 2 / \ldots / \mathrm{LJ} / / \mathrm{I} 2 / \mathrm{L} 1 / \ldots / \mathrm{LJ} / / \ldots
$$

where a given stimulus ends with a double carriage return after the last line.

To maximize the speed of stimulus presentation, an additional parameter (number of lines) is entered as the first byte of a given stimulus array element. This parameter allows the trials program to present the multiple-line stimulus without using the lengthy Monitor routine COUT.

The required modifications to the stimulus input loop of the first BASIC program are given in Appendix D. (Lines 280-360 of Appendix A are replaced as indicated.) For a given stimulus, the array address corresponding to the number of lines is $\mathbf{N}$ and that of the length of the current line is LS. Both N and LS must be real variables. Individual stimulus characters are obtained from the stimulus file using the GET command.

If a given character is not a carriage return, LS is incremented and the character is POKEd into the array. If a given character is a carriage return, LS will not be incremented. Therefore, LS will not be zero if a carriage return represents the end of a stimulus line, as opposed to the end of the stimulus proper. In this case, the number of lines is incremented (Line 322) and LS is established for the next line. However, LS is zero when the 
carriage return is the second of a pair of carriage returns. At this point, garbage collection is forced. After the GET command is used, a carriage return must be printed before any DOS command is recognized (Line 360).

The changes in the trials program are shown in Appendix E. The line numbers in the listing are arbitrary. The changes are confined to the STIMON and CLR sequences. The program makes use of the Monitor routine BASCALC. This routine is called with a screen line number in the A-register. It calculates the columnzero screen address corresponding to the line number and places that address in the pointers BASL/BASH. The program keeps a record of the current line number at $\mathrm{CV}$.

In presenting a given stimulus, the starting line is initialized by a JSR to BASCALC. The number of lines is indexed by a zero-page location. At label STIMON1, each character of a given line is placed on the screen. After a line is printed, and if it is not the last line of the stimulus, the current line number is incremented (twice for a double space) and BASCALC is called. Then the next line is printed.

When a stimulus is to be cleared from the screen, subroutine CLR is called. A constant number of lines is always cleared.

Minor changes must be made to the second BASIC program (Appendix B). The changes are related to the use of the GET command to skip over the stimulus characters while the ID numbers are being obtained for the data file. The replacement lines are as follows:

\section{FOR I = 1 TO P: INPUT ID: GOSUB 900: NEXT}

260 GOSUB 900

280 PRINT CHRS (13);D\$ "CLOSE"

900 GET $\$ \$$ : IF $S \$<>$ CHR\$ (13) THEN 900

910 GET S\$: IF S\$ < > CHR\$ (13) THEN 900

920 RETURN

\section{PRECISION AND STIMULUS CAPACITY}

In the illustrated application, and in all mentioned single-line applications, each individual operation of the trials program, such as presenting the stimulus, takes less than $1 \mathrm{msec}$ to perform. Therefore, millisecond precision of program operations, both within and across trials, is maintained in these experiments. However, in multipleline experiments, the time required to present the stimulus can exceed $1 \mathrm{msec}$ due to absolute speed limitations imposed by the hardware. The time to present a given stimulus depends on the number of lines. If an average line is considered to be 37 characters (the first BASIC program does not complete partial lines with blanks), and if an average half line is considered to be 20 characters, then a $11 / 2$ line stimulus is presented in $1.5 \mathrm{msec}$, and a $21 / 2$ line stimulus is presented in $2.3 \mathrm{msec}$. These times are based on $9.78 \mathrm{E}-4 \mathrm{msec}$ per machine cycle (approximately $1 \mathrm{MHz}$ ).
Stimulus capacity depends on the elaborateness of the first BASIC program. For the illustrated program (Appendix A), there are approximately $29 \mathrm{~K}$ free bytes after the compiled program is loaded. This corresponds to a storage space for 2,975 centered words of seven letters each, or 959 uncentered lines of 30 characters each. For a multiple-line reading experiment, incorporating the changes in Appendix D, the number of free bytes is very similar. This results in storage space for 497 sentences of $1 \frac{1}{2}$ lines each (average stimulus lines as defined previously).

As a comparison, tests were carried out using the conventional array method. A single compiled BASIC program was written that used Applesoft arrays for ID numbers, stimuli, responses, and latencies. The program read duplicate sets of array elements from a long file until capacity was reached. Dimension statements were adjusted as necessary to maximize capacity. The following limits were observed: 1,713 centered words of seven letters each; 790 uncentered lines of 30 characters each; and 446 sentences of $1 \frac{1}{2}$ lines each. The ID numbers were always four digits. Each latency was three digits in the centeredword test and four digits in the other two tests. The results indicate that the capacity advantage of the illustrated method over the conventional one is most pronounced when the ratio of data characters to stimulus characters is relatively high, which occurs when the individual stimuli are short. The advantage is due primarily to the overlaying of arrays.

The programming method outlined here may be applied to a wide variety of experimental paradigms that use visually-presented verbal stimuli. The method is useful when maximum precision and stimulus capacity are required.

\section{REFERENCES}

Apple Computer, Inc. (1981). Apple II Reference Manual. Cupertino, CA: Author.

Collins, A. M. \& Quillian, M. R. (1969). Retrieval time from semantic memory. Journal of Verbal Learning \& Verbal Behavior, 8, 240-247.

DLhopolsky, J. G. (1983). Limitations of high-level microcomputer languages in software designed for psychological experimentation. Behavior Research Methods \& Instrumentation, 15, 459-464.

Glanzer, M. , Dorfman, D. , Kaplan, B. (1981). Short-term storage in the processing of text. Journal of Verbal Learning \& Verbal Behavior, 20, 656-670.

HoCKLEY, W. E. (1982). Retrieval processes in continuous recognition. Journal of Experimental Psychology: Learning, Memory, \& Cognition, 8, 497-512.

NEELY, J. H. (1977). Semantic priming and retrieval from lexical memory: Roles of inhibitionless spreading activation and limitedcapacity attention. Journal of Experimental Psychology: General, 106, 226-254.

RAYFIELD, F., \& CARNEY, J. (1981). Controlling behavior experiments with BASIC on 6502-based microcomputers. Behavior Research Methods \& Instrumentation, 13, 735-740.

REED, A. V. (1979). Microcomputer display timing: Problems and solutions. Behavior Research Methods \& Instrumentation, 11, 572-576.

Rubenstein, H., Garfield, L., \& Millikan, J. A. (1970). Homographic entries in the internal lexicon. Journal of Verbal Learning \& Verbal Behavior, 9, 487-494. 


\section{NOTES}

1. The model is "The Clock" and is available from Mountain Computer Incorporated, 300 El Pueblo, Scotts Valley, CA 95066. The current retail price is $\$ 280$.
2. Available from Microsoft Consumer Products, 400108 th Avenue NE, Suite 200 , Bellevue, WA 98004 . The current retail price is $\$ 180$ (\$118 discount).

3. Program listings (and source code if a floppy disc is provided) are available from the author at no charge.

APPENDIX A

Listing of First BASIC Program (Lexical Decision)

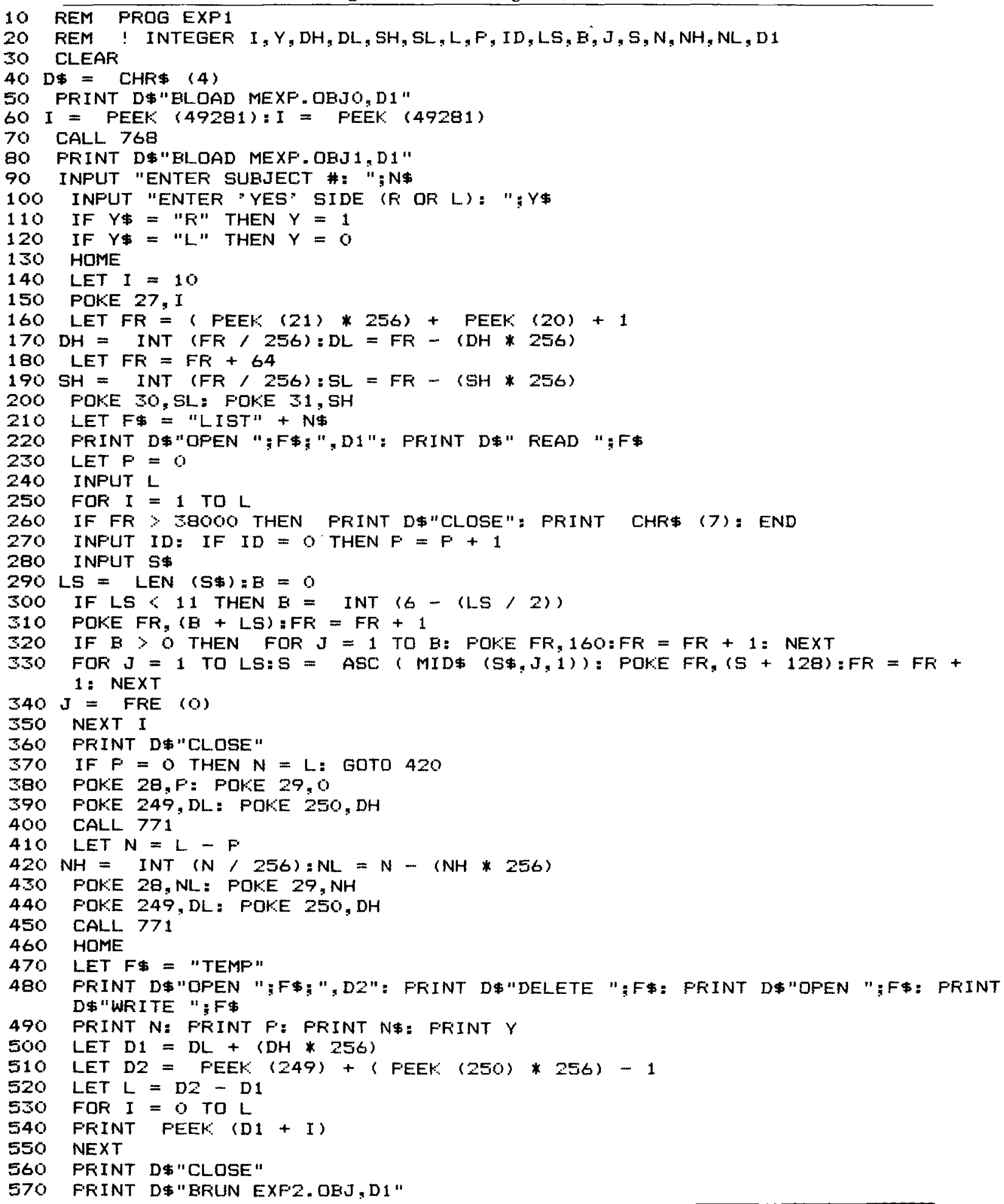


APPENDIX B

Listing of Second BASIC Program (Lexical Decision)

10

20

30

40

$50 \mathrm{D}$

60

70 FFINT D\$"OPEN

BO INFUT N. INFUT F. INFUT N\$. INPUT Y

90 FOR I $=1$ TO $\mathrm{N}$

100 INFUT R(I)

110 INFUT TL

120 INFUT TH

$130 \mathrm{FT}(\mathrm{I})=(\mathrm{TH} * 256)+\mathrm{TL}$

140 IF $Y=0$ AND $F(I)=0$ THEN $R(I)=1:$ GOTO 160

150 IF $Y=0$ AND $F(I)=1$ THEN $F(I)=0$

160 NEXT

170 FFINT D\$"CLOSE"

180 LET F\$ = "LIST" + N\$

190 FRINT D\&"OFEN "\#F\$" DI": FRINT D\$"READ "F\$

200 INFUT $L$

210 IF $F=0$ THEN 240

220 LET $L=L-F$

230

240

250

260

270

280

290

300

310

320

330

340

350

360

370

380

390

FOF $I=1$ TO F: INFUT ID: INFUT S\$: NEXT

FOF $I=1$ TO L

INFUT ID(I): IF ID(I) $\Rightarrow 8999$ THEN $N=N-1$

INFUT S\$

NEXT

FRINT D\$"CLOSE"

LET F\$ = "DATA" + N\$ DW"WRITE "F\$

FRINT N

FOF $I=1$ TO L

IF ID(I) $>8999$ THEN 370

FFINT ID(I)

FFINT Fi (I)

FRIINT RTT (I)

NEXT

FRINT D\$"CLOSE"

FRINT D\$"BRUN SUMMARY. OBJ, D 1 "

FRINT D\$"OFEN ":F\$;",D2": FRINT D\$"DELETE ":F\$: FRINT D\$"OFEN ";F\$: PRINT

APPENDIX C

The Page-3 and Trials Programs (Lexical Decision)

SOUFCE FILE: TMF'

\begin{tabular}{|c|c|c|c|c|}
\hline $0006:$ & 1 & $\mathrm{TL}$ & ERU & $\$ 06$ \\
\hline $0007:$ & 2 & $\mathrm{TH}$ & EQU & \$07 \\
\hline $001 A:$ & 3 & TEMF & EQU & $\$ 1 \mathrm{~A}$ \\
\hline $001 \mathrm{E}:$ & 4 & ISI & EQU & D. $1 \mathrm{~B}$ \\
\hline $0010:$ & 5 & NL & EOL & $\$ 1 C$ \\
\hline $001 \mathrm{D}:$ & 6 & $\mathrm{NH}$ & EQU & \$ $1 \mathrm{D}$ \\
\hline $001 E:$ & 7 & $S L$ & EQU & D1E \\
\hline OO1F: & a & SH & EOU & $\$ 1 F$ \\
\hline OOFS: & 9 & DL & EQLI & DFG \\
\hline OOFA: & 10 & $\mathrm{DH}$ & EQU & DFA \\
\hline $0534=$ & 11 & SCF & EQU & $\$ 0554$ \\
\hline $\cos 1:$ & 12 & LEFT & EQU & $\$ 0061$ \\
\hline $0062:$ & 13 & $M I D$ & EQU & $\$ C 062$ \\
\hline $\operatorname{COE} 3:$ & 14 & FIIGHT & EDU & 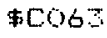 \\
\hline Coc4: & 15 & CL_OCF: & EQU & $\$ \operatorname{coc} 4$ \\
\hline
\end{tabular}

:LSE FT (TICKS $\times 1$ )

MSE RT (TICKS X 25S)

1-EYTE TEMF STORE

\# 50 MSEC ELKS ISI

LSE \# TFIIALS

MSE " "

"LSE STIM FOINTEF'

"MSE " "

LSB DATA FOINTEF

"MSE "SCFEEN (LINE 10, COL 12)

LEFT RESF FOFT

STAFT FOFT

FIGHT RESF FOFT

; 1 MSEC CLOCK ROM 


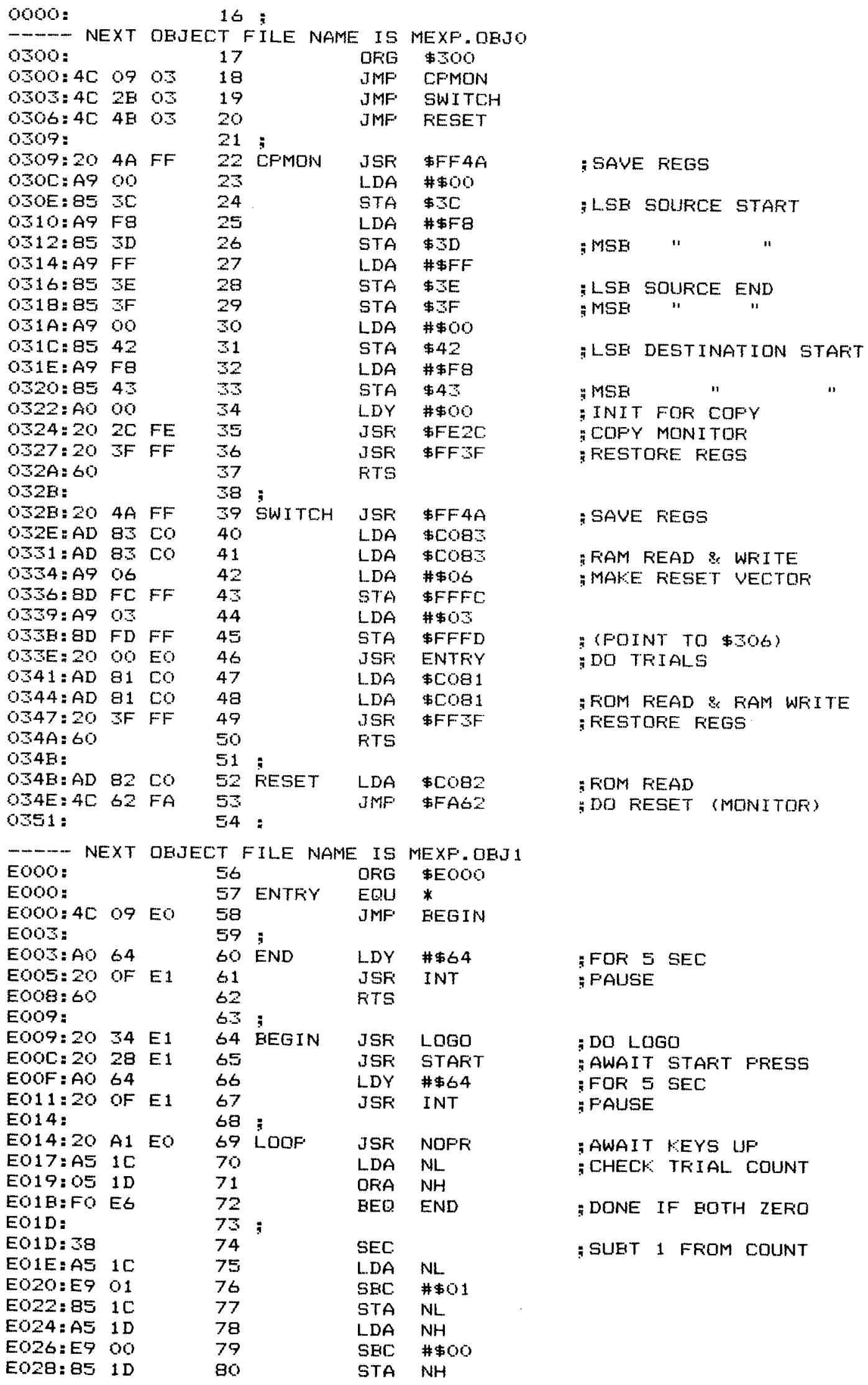




\begin{tabular}{|c|c|c|c|c|c|c|c|}
\hline EOLA: & & & 81 & 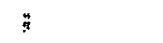 & & & \\
\hline EO2A: $A O$ & 00 & & 82 & & LDY & $\# \$ 00$ & ; INIT \\
\hline$E O 2 C=\mathrm{BI}$ & $1 E$ & & 83 & & LDA & $(S L), Y$ & :STIM LENGTH \\
\hline$E O 2 E: A A$ & & & 84 & & TAX & & \\
\hline$E O 2 F: C A$ & & & 95 & STIMON & DEX & & \\
\hline $\mathrm{EOBO}: \mathrm{Cg}$ & & & 86 & & INY & & \\
\hline$E 031: E 1$ & 1E & & 87 & & LDA & $(S L), Y$ & GET CHAF \\
\hline$E 033: 99$ & 34 & 05 & 98 & & STA & $S C F, Y$ & FUT ON SCFEEN \\
\hline EOSB:EO & 00 & & 89 & & CFX & $\# \$ 00$ & ¿ OUUTFUT DONE? \\
\hline$E 038: 00$ & $F E$ & & 90 & & BNE & STIMON & NO? DO ANOTHEF CHAF \\
\hline EOSA: & & & 91 & $\vdots$ & & & \\
\hline EOBA:20 & D6 & EO & 92 & & JSF & UPDATE & ;FOINT TO NEXT STIM LENGTH \\
\hline EOSD: & & & 93 & ; & & & \\
\hline$E O B D: A D$ & $\mathrm{C4}$ & $\mathrm{co}$ & 94 & & LDA & CLOCK & FEAD TIME \\
\hline $\mathrm{EO40:85}$ & $1 \mathrm{~A}$ & & 95 & & STA & TEMF & MAYE FEFEFENCE \\
\hline$E 042: A 9$ & 00 & & 96 & & $1 \mathrm{DA}$ & $\# \$ 00$ & \\
\hline E044:85 & 06 & & 97 & & STA & $T L$ & \\
\hline E046:85 & 07 & & 98 & & STA & TH & INITS \\
\hline EO4B: & & & 99 & 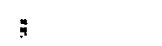 & & & \\
\hline$E 049: A O$ & 01 & & 100 & PCHECK & LDY & $\# 01$ & $Y Y=1$ FOFI FIEHT \\
\hline$E 04 A=2 \mathrm{C}$ & 63 & $\mathrm{CO}$ & 101 & & EIT & FIGHT & YEYFFESS? \\
\hline$E O 4 D: 10$ & 32 & & 102 & & BF'L & FTCHK & YES? \\
\hline$E 04 F: B 8$ & & & 103 & & DEY & & $Y Y=0$ FOF LEFT \\
\hline $\mathrm{EOSO}=2 \mathrm{C}$ & 61 & $\mathrm{CO}$ & 104 & & BIT & LEFT & KEYFRESS? \\
\hline EOSS: 10 & $2 \mathrm{C}$ & & 105 & & $\mathrm{EF}-\mathrm{L}$ & FITCHE & YES? \\
\hline EOS5: & & & 100 & 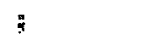 & & & \\
\hline E055: & & & 108 & 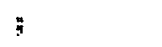 & & & \\
\hline EO55:AD & {$[4$} & Co & 109 & & $1 . D A$ & CLOCF & STIME CHECK \\
\hline EO58: $\mathrm{CE}$ & $1 \mathrm{~A}$ & & 110 & & CMF & TEMF' & HHAS CLDCK TICKED? \\
\hline$E O 5 A=F O$ & $\mathrm{EC}$ & & 111 & & BEQ & FCHECF & SNO? CHECK FORTS \\
\hline EOSC: 85 & $1 \mathrm{~A}$ & & 112 & & STA & TEMF & ; YES? UFDATE REFERENCE \\
\hline$E O S E: 18$ & & & 113 & & CLC: & & \& ADD 1 MSEC TO FT \\
\hline EOSF:AS & 06 & & 114 & & $\operatorname{LDA}$ & $\mathrm{TL}$ & \\
\hline$E 061: 69$ & OI & & 115 & & $A D C$ & $\# \$ 01$ & \\
\hline EOSE: 85 & 06 & & 116 & & STA & $\mathrm{TL}$ & \\
\hline$E 065: A 5$ & 07 & & 117 & & LDA & $\mathrm{TH}$ & \\
\hline$E 067: 69$ & 00 & & 118 & & $A D C$ & $\# \$ 00$ & \\
\hline$E 069: 85$ & 07 & & 119 & & STA & $\mathrm{TH}$ & \\
\hline$E 06 B=E 9$ & 76 & & 120 & & CMF & $\# \$ 76$ & ¿FT AT 30,208 MSEL MAX? \\
\hline$E O G D=F O$ & 03 & & 121 & & EEQ & NOFIESF & YES? FLAG \\
\hline$E O G F: 4 C$ & 48 & EO & 122 & & JMF & FCHECK & \#O? AWAIT RESFUNSE \\
\hline EO72: & & & 123 & 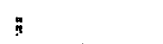 & & & \\
\hline$E 072: A 0$ & 65 & & 124 & NOFESF & LDY & \#\$63 & ;FLAG (DECIMAL 99) \\
\hline$E 074: 2 \mathrm{C}$ & 61 & C.0 & 125 & NDFESF' 1 & EIT & LEFT & AWAIT KEYFFESS \\
\hline$E 077: 10$ & 18 & & 126 & & BFL. & FSAVE & \& RECOFD \\
\hline$E 079: 2 C$ & $6 \div$ & $\mathrm{Co}$ & 127 & & EIT & RIGHT & \\
\hline$E 07 C: 10$ & 13 & & 128 & & EFL. & FSAVE & \\
\hline$E O 7 E: 4 C$ & 74 & $E$ & 129 & & $\mathrm{JMF}$ & NORESF 1 & \\
\hline EO81: & & & 130 & 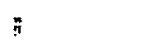 & & & \\
\hline EOB1:A5 & 07 & & 151 & FTCHK & LDA & TH & : KEYBOUNCE? \\
\hline EOGS:DO & OC & & 132 & & ENVE & FSAVE & NNOT IF MSE $\because 0$ \\
\hline EOBS:AS & 06 & & 13 & & LDA & TL & \\
\hline$E 087: C 9$ & 80 & & $1 \Xi 4$ & & CMF & $\# \$ 80$ & \\
\hline$E 089: 10$ & 06 & & 135 & & EFL & RSAVE & NOT IF LSE $\because$ DECIMAL 127 \\
\hline EOAE: 20 & $A C$ & EO & 136 & & JSF & TNOFF & YES? DO TIMED NOFFESS \\
\hline EOBE: $4 C$ & 48 & $\mathrm{EO}$ & 157 & & JMF & FCHECK & THEN AWAIT NEW FESF \\
\hline$E 091: 84$ & $1 \mathrm{~A}$ & & 158 & FSAVE & STY & TEMF & T TEMF SAVE \\
\hline E09:: & & & 1.59 & 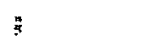 & & & \\
\hline$E 095: 20$ & E4 & EO & 140 & & JSFi & CLF & aLEAF SCFEEN \\
\hline E096: & & & 141 & $\vdots$ & & & \\
\hline E096: 20 & $F_{1}$ & EO & 142 & & JSF & SAVDAT & S SAVE RESF \& FIT \\
\hline E099: & & & $14 \Xi$ & ; & & & \\
\hline$E 099: A 4$ & $1 \mathrm{~B}$ & & 144 & & LDY & ISI & \# 50 MSEC ELKS \\
\hline EOQE: 20 & $\mathrm{OF}$ & $E 1$ & $\begin{array}{l}145 \\
146\end{array}$ & & JSF & INT & :TIME ISI \\
\hline
\end{tabular}




\begin{tabular}{|c|c|c|c|c|c|c|c|}
\hline EOPE: $4 C$ & 14 & EO & 147 & & $J M F$ & LOOF & EOTTOM OF LOOF \\
\hline EOA1: & & & 148 & $;$ & & & \\
\hline EOA1: & & & 149 & 3 & & & \\
\hline EOA1: & & & 151 & SUEFGUT & TINES: & & \\
\hline EOA1: & & & 152 & 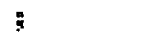 & & & \\
\hline EOA1: & & & 153 & 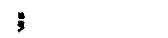 & & & \\
\hline$E O A 1: 2 C$ & 61 & 20 & 154 & NOFF & EIT & LEFT & \\
\hline$E O A 4: 10$ & $\mathrm{FE}$ & & 155 & & EFL & NOFF & \#WAIT FOF NOFRESS \\
\hline$E O A B: 2 C$ & 63 & $\mathrm{CO}$ & 156 & & EIT & FIGHT & \\
\hline EOA9: 10 & FG & & 157 & & EF'L & NOFF & ;WAT FOR NOFFESS \\
\hline EOAE: 60 & & & 158 & & FTS & & \\
\hline EOAC: & & & 159 & 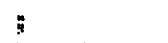 & & & \\
\hline$E O A C: A D$ & C4 & $\mathrm{CO}$ & 160 & TNOFF & L.DA & CLOCK & \\
\hline$E O A F=C E$ & $1 \mathrm{~A}$ & & 161 & & CMF & TEMF & \#HAS CLDCK TICKED? \\
\hline $\mathrm{EOB1}=\mathrm{FO}$ & 18 & & 162 & & BEQ & TNOFF1 & NO? DO NOFFIESS \\
\hline EOEZ: 85 & $1 \mathrm{~A}$ & & 163 & & STA & TEMF & YES? UFDATE REF \\
\hline EOE5: 18 & & & 164 & & CLC & & ADD 1 MSEC TO FT \\
\hline EOEG: $A 5$ & 06 & & 165 & & LDA & $T L$ & ( \\
\hline EOB8: 69 & 01 & & 166 & & $A D C$ & $\#$ \#01 & \\
\hline EOEA: 85 & ot & & 167 & & STA & TL & \\
\hline EOEC: AS & 07 & & 168 & & $\angle D A$ & $\mathrm{TH}$ & \\
\hline EOBE: 69 & 00 & & 169 & & $A D C$ & $\# \$ 00$ & \\
\hline EOCO: $\mathrm{BD}$ & 07 & & 170 & & STA & $\mathrm{TH}$ & \\
\hline $\mathrm{EOC2}=\mathrm{C9}$ & 76 & & 171 & & CMF & \#\$76 & ¿ET AT 30,20Q MSEC MAX? \\
\hline $\mathrm{EOC} 4: \mathrm{DO}$ & 05 & & 172 & & ENE & TNOFR 1 & NOP DO NOFRESS \\
\hline EOC6: 68 & & & 173 & & FLA & & YES? \\
\hline EOC7: 68 & & & 174 & & FLA & & SKIIP USUAL FETUFN \\
\hline EOCB: $4 \mathrm{C}$ & 72 & EO & 175 & & $J_{M} M^{r}$ & NOFESF & ; FLAG \\
\hline EOCE: $2 \mathrm{C}$ & 61 & $\mathrm{CO}$ & 176 & TNOF'R 1 & EI T & LEFT & LIFE NOPF \\
\hline EOCE: 10 & $\mathrm{DC}$ & & 177 & & EFL & TNOFF & \\
\hline EODO: $2 \mathrm{C}$ & 63 & $\mathrm{co}$ & 178 & & EIT & FIGHT & \\
\hline EODS: 10 & 07 & & 179 & & EF'L & TNOFF & \\
\hline EODS: 60 & & & 180 & & FITS & & \\
\hline EODG: & & & 181 & 3 & & & \\
\hline EODG: $\mathrm{CB}$ & & & 182 & UPDATE & INY & & \\
\hline EOD7: 98 & & & 183 & & TYA & & GET INCR \\
\hline EODE: 18 & & & 184 & & CLC & & ADD TD $S L / S H$ \\
\hline EOD9: 65 & $1 E$ & & 195 & & $A D C$ & SL & \\
\hline EODE: 85 & $1 E$ & & 186 & & STA & SL & \\
\hline$E O D D=A S$ & $1 F$ & & 187 & & LDA & $\mathrm{SH}$ & \\
\hline EODF : 69 & $\infty$ & & 188 & & $A D C$ & $\# \$ 00$ & \\
\hline EOE1 : 85 & $1 F$ & & 189 & & STA & SH & \\
\hline EOES: 60 & & & 190 & & ETS & & \\
\hline EOE4: & & & 191 & 3 & & & \\
\hline EOE4: AO & 00 & & 192 & CLFF & LDY & $\# \$ 00$ & INIT \\
\hline EOEG: A9 & AO & & 193 & & LDA & $\# \$ A O$ & BLANK: \\
\hline EOEB: CB & & & 194 & CLR 1 & INY & & \\
\hline EOE9: 99 & 34 & 05 & 195 & & STA & SCF, $Y$ & FRINT ELANK \\
\hline EOEC: $\mathrm{CO}$ & $O F$ & & 196 & & $C F Y$ & \#\$OF & DONE $15 ?$ \\
\hline EOEE : DO & $\mathrm{Fg}$ & & 197 & & ENE & CLR1 & NO? DO ANDTHER \\
\hline EOFO: 60 & & & 198 & & RTS & & \\
\hline $\begin{array}{l}\text { EOF 1: } \\
\text { EOF 1: }\end{array}$ & & & $\begin{array}{l}199 \\
201\end{array}$ & 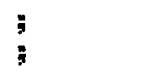 & & & \\
\hline$E O F 1=A O$ & 00 & & 202 & SAVDAT & LDY & $\# \$ 00$ & INIT \\
\hline EOFS: A5 & $1 \mathrm{~A}$ & & 203 & & $\angle D A$ & TEMP & \\
\hline EOF5: 91 & F9 & & 204 & & STA & $(D L), Y$ & :STORE RESF FIRST \\
\hline EOF7: $\mathrm{CB}$ & & & 205 & & INY & & \\
\hline EOFB: A5 & Ob & & 206 & & LDA & TL & \\
\hline EOFA: 91 & $F 9$ & & 207 & & STA & $(D L), Y$ & THEN LSE FT \\
\hline EOFC : CB & & & 208 & & INY & & \\
\hline EOFD:AS & 07 & & 209 & & LDA & TH & \\
\hline EOFF: 91 & Fq & & 210 & & STA & $(D L), Y$ & THEN MSE FT \\
\hline $\mathrm{E} 101: \mathrm{CB}$ & & & 211 & & $I N Y$ & & \\
\hline E102:98 & & & 212 & & TYA & & GET INCR \\
\hline$E 103: 18$ & & & 213 & & CLC & & \ADD TO DL/DH \\
\hline$E 104: 65$ & $F 9$ & & 214 & & $A D C$ & $\mathrm{DL}$ & \\
\hline
\end{tabular}




\begin{tabular}{|c|c|c|c|c|c|c|}
\hline E106:85 & $\mathrm{FQ}$ & & 215 & & STA & $\mathrm{DL}$ \\
\hline$E 108: A 5$ & FA & & 216 & & LDA & $\mathrm{DH}$ \\
\hline E10A: 69 & 0 & & 217 & & ADC & $\# 100$ \\
\hline$E 10 C=85$ & FA & & 218 & & STA & $\mathrm{DH}$ \\
\hline E1OE: 60 & & & 219 & & FITS & \\
\hline E1OF: & & & 220 & 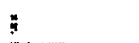 & & \\
\hline$E 1 O F=88$ & & & 221 & INT & $D E Y$ & \\
\hline$E 110: A 2$ & 32 & & 222 & & $\operatorname{LDX}$ & $\# \$ 32$ \\
\hline$E 112=C A$ & & & 223 & INT I & DEX & \\
\hline E113:AD & C4 & $\mathrm{CO}$ & 224 & & LDA & CLOCK \\
\hline E116:85 & $1 \mathrm{~A}$ & & 225 & & STA & TEMF \\
\hline$E 118: A D$ & C4 & $\mathrm{CO}$ & 226 & INT2 & LDA & CLOCF \\
\hline$E 11 \mathrm{~B}: \mathrm{CS}$ & $1 A$ & & 227 & & CMF & TEMP \\
\hline$E 11 \mathrm{D}: \mathrm{FO}$ & Fq & & 228 & & EEQ & INT2 \\
\hline$E 11 \mathrm{~F}: E O$ & 00 & & 229 & & CFX & $\# \$ 00$ \\
\hline$E 121: D 0$ & $E F$ & & 230 & & ENE & INT 1 \\
\hline$E 123: C O$ & $\phi$ & & 251 & & $C F Y$ & $\# \$ 00$ \\
\hline$E 125: D 0$ & $E \theta$ & & 232 & & ENE & INT \\
\hline E127:60 & & & 235 & & FITS & \\
\hline E128 & & & 234 & I & & \\
\hline$E 128: 2 C$ & 62 & $\mathrm{Co}$ & 235 & STAFT & EIT & MID \\
\hline E12B:10 & 03 & & 236 & & BFL & STAFT \\
\hline$E 12 D: 4 C$ & 28 & $E 1$ & 237 & & JMF & START \\
\hline$E 130: 20$ & E4 & EO & 238 & STAFT 1 & $\sqrt{S F}$ & CLF \\
\hline E15: :60 & & & 239 & & RTS & \\
\hline E1:4: & & & 240 & 3 & & \\
\hline$E 154: A O$ & 00 & & 241 & LOGO & L.DY & $\# \$ 00$ \\
\hline E156:A2 & 05 & & 242 & & $1 . D x$ & $\# \$ 05$ \\
\hline$E 1=8: A 9$ & $A A$ & & 243 & & L.DA & $\#$ \#AA \\
\hline$E 13 A=C A$ & & & 244 & LOGO 1 & DEX & \\
\hline$E 1 \leq E: C 8$ & & & 245 & & INY & \\
\hline$E 1 \mathrm{IC}: \mathrm{CB}$ & & & 246 & & INY & \\
\hline$E \perp 50=99$ & 34 & 05 & 247 & & STA & SCF, $Y$ \\
\hline$E 140=E O$ & 00 & & $24 \theta$ & & CFX & \#\$00 \\
\hline$E 142=00$ & FG & & 249 & & ENE & LOGOL \\
\hline$E 144: 60$ & & & 250 & & FITS & \\
\hline
\end{tabular}

START I ELF.

$\because F O F$ SO MSEC START 1 MSEC FIEAD TIME - MAKE REFEFIENCE

VALLUE CHANGED? NO? READ AGAIN BLK DONE? - NO? DO ANOTHEF MSEC ALL ELKS DONE? -NO? DO ANOTHER BLKK

HEYFFESS?

YES? GD NO? CHECY AGAIN "CLEAF SCFEEN

INITS

- ASTERISK

: (LEAVE FLANk: BETWEEN) $\because$ FUT ON SCFEEN ; DONE 5? $\because N O ?$

*** SUCCESSFUL ASSEMELY Y NO EFFIOFS

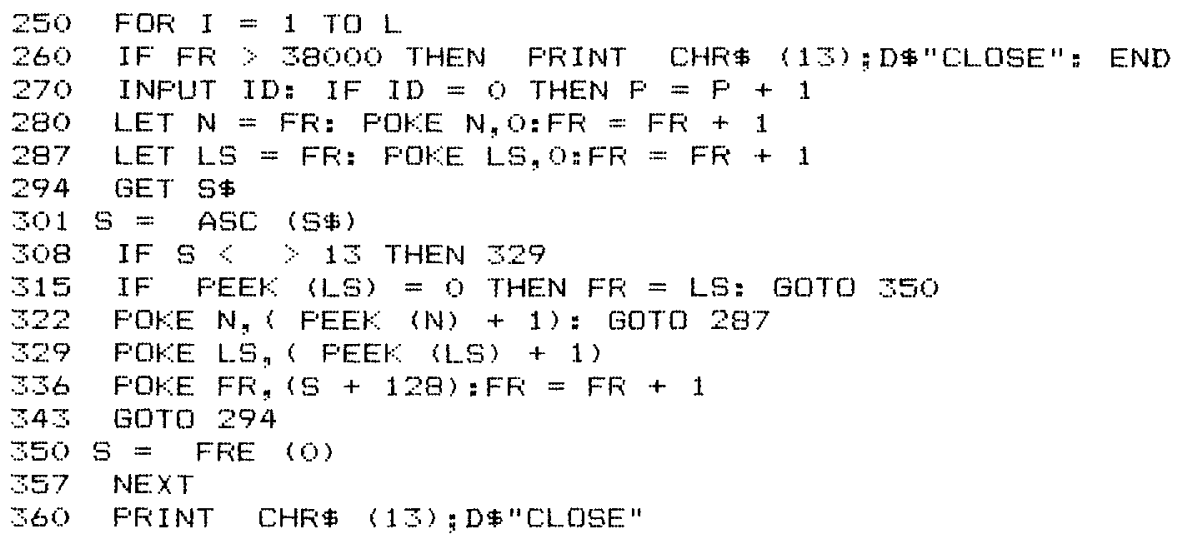


APPENDIX E

Replacement Sequences for Trials Program (Multiple-Line Reading)

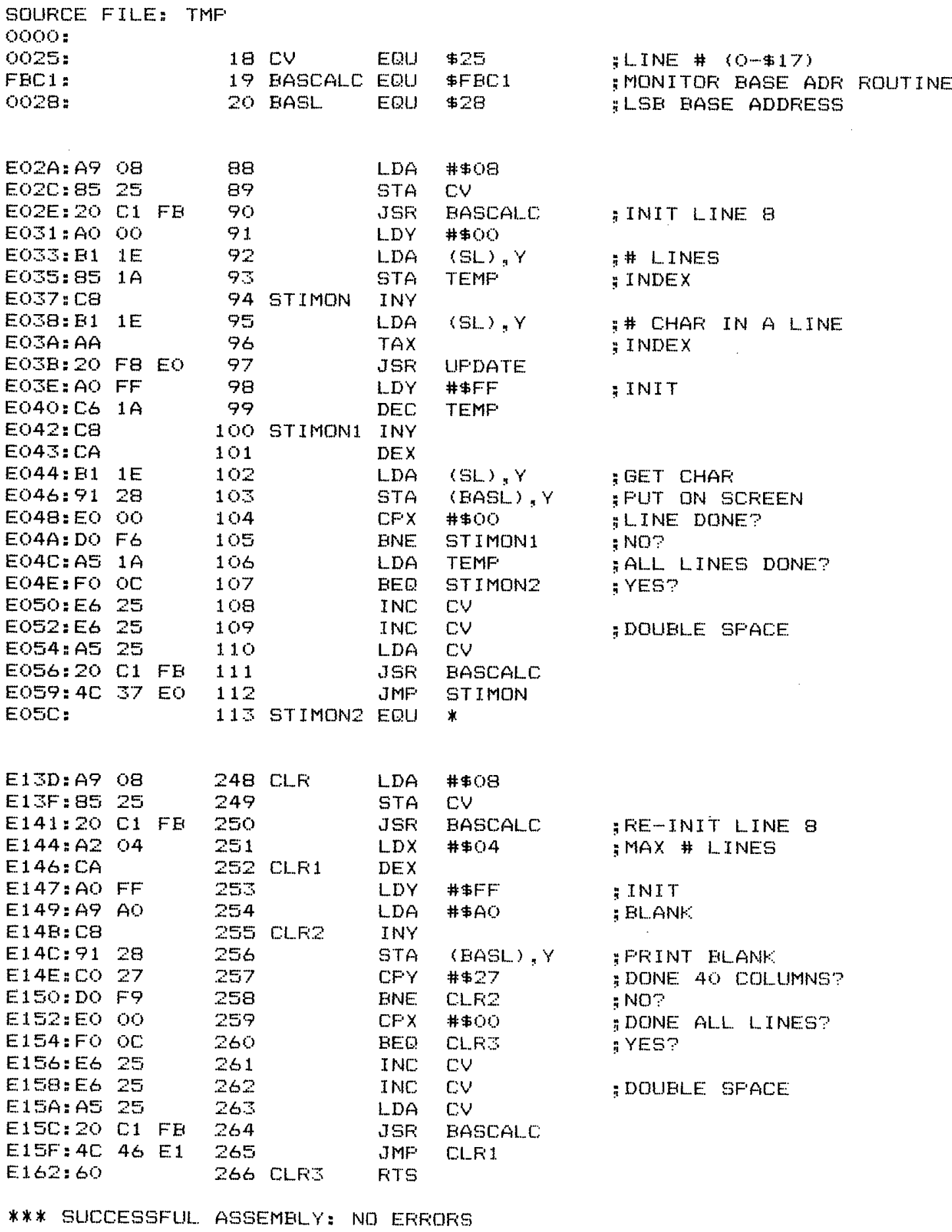

